OPEN ACCESS

Edited by:

Lionel Apetoh,

Institut National de la Santé et de la

Recherche Médicale (INSERM),

France

Reviewed by:

Adam Snook,

Thomas Jefferson University, United States

Fernando Aranda,

August Pi i Sunyer Biomedical

Research Institute (IDIBAPS), Spain

*Correspondence:

Venkataswarup Tiriveedhi

vtirivee@tnstate.edu

Specialty section:

This article was submitted to Women's Cancer,

a section of the journal

Frontiers in Oncology

Received: 30 January 2019 Accepted: 08 April 2019 Published: 25 April 2019

Citation:

Khandekar D, Amara S and Tiriveedhi V (2019) Immunogenicity of Tumor Initiating Stem Cells: Potential Applications in Novel Anticancer Therapy. Front. Oncol. 9:315. doi: 10.3389/fonc.2019.00315

\section{Immunogenicity of Tumor Initiating Stem Cells: Potential Applications in Novel Anticancer Therapy}

\author{
Durga Khandekar ${ }^{1}$, Suneetha Amara ${ }^{2}$ and Venkataswarup Tiriveedhi ${ }^{1,3 *}$ \\ ${ }^{1}$ Department of Biological Sciences, Tennessee State University, Nashville, TN, United States, ${ }^{2}$ Department of Medicine, St. \\ Thomas Hospital-Midtown, Nashville, TN, United States, ${ }^{3}$ Department of Pharmacology, Vanderbilt University, Nashville, TN, \\ United States
}

Tumor initiating stem cells (TISCs) are a subset of tumor cells, which are implicated in cancer relapse and resistance to chemotherapy. The metabolic programs that drive TISC functions are exquisitely unique and finely-tuned by various oncogene-driven transcription factors to facilitate pro-cancerous adaptive challenges. While this change in TISC metabolic machinery allows for the identification of associated molecular targets with diagnostic and prognostic value, these molecules also have a potential immunological application. Recent studies have shown that these TISC-associated molecules have strong antigenic properties enabling naïve $C D 8+T$ Iymphocytes to differentiate into cytotoxic effector phenotype with anticancer potential. In spite of the current challenges, a detailed understanding in this direction offers an immense immunotherapeutic opportunity. In this review, we highlight the molecular targets that characterize TISCs, the metabolic landscape of TISCs, potential antitumor immune cell activation, and the opportunities and challenges they present in the development of new cancer therapeutics.

\section{Keywords: antigens, antibodies, breast cancer, tumor initiating cells, vaccines, immunotherapy}

\section{INTRODUCTION}

Drug resistance remains a major challenge in long-term therapeutic success of cancer patients (1). Current anticancer therapies target rapidly dividing cells with the assumption that cancer cells divide at a rate 100-1000 times higher than the normal terminally differentiated cells. Considerable debate exists to explain for the reason behind acquiring the tumorigenicity and heterogeneity of otherwise normal cells. Two non-mutually exclusive theories are well acknowledged in the cancer research field to explain for the tumor heterogeneity $(2,3)$. One theory proposes the idea that a normal cell becomes a cancer cell due to an acquired or inherited genetic change (referred to as a mutation) giving rise to a clone of cancerous cells. This clone further accumulates genetic changes from environmental or other injuries (second-hit) leading to the evolution of multiple clones, of which, only a few, due to selection pressure and/or due to their ability to escape host immune destruction, ultimately develop into full-fledged cancer. This selection pressure on multiple subclones explains for the tumor heterogeneity (4). Yet another theory, advances an idea that tumors arise from otherwise quiescent progenitor stem cells with a cancer-initiating capability (referred to as tumor initiating stem cells [TISCs]) and upon appropriate external stimulus or an epigenetic change a subset of these stem cells transform to develop full cancers. This proliferative ability leads to expression of markers at various phases of the progression of the stem cells and thus 
explaining for the final tumor heterogeneity (5). TISCs have been identified in several cancers including breast cancer, brain cancer, and colon cancer (6). TISCs are considered to be resistant to standard chemotherapeutic regimens and play an important role in cancer-relapse. As most of the chemotherapeutic agents target rapidly dividing cells they have minimal effect on quiescent TISCs. From cancer immunotherapy perspective, it is important to note that TISCs display unique heterogeneity with expression of TISC-associated mutated or overexpressed protein commonly referred to as TISC-associated antigens, which open new venues of anti-cancer immunotherapy. Further, TISCs have a distinct metabolic phenotype which leads to overexpression of certain enzymes which could also be utilized for the development of targeted effector immune response. In this review, we will discuss the metabolic phenotypes and molecular suigeneris related with TISCs, and then, discuss the possible application of these molecular targets in the development of vaccine and cell based anti-cancer immunotherapeutic tools.

\section{PHENOTYPIC DIFFERENCES BETWEEN NORMAL STEM CELLS AND TISCS}

While normal stem cells ([NSCs], such as embryonic stem cells [ESC] and hematopoietic progenitor cells) and TISCs have certain similarities, in that both have the ability to self-renew and differentiate into various organ with histological features, yet, they both have differences in various genetic, morphological and phenotypic features (7). Specifically, there is a stark contrast in the mitochondrial features between NSCs and TISCs, in that mitochondria of NSCs have a lower DNA copy number, poorly developed morphology, and minimal oxidative phosphorylation (OXPHOS) capacity. In contrast, TISCs display increased mitochondrial mass and mitochondrial biogenesis (8). In spite of an increased number of mitochondria, TISCs have been attributed with enhanced glycolytic phenotype, while, terminally differentiated cells were considered to rely mostly on oxidative phosphorylation (OXPHOS) $(9,10)$ for ATP production. Along with upregulation of glycolysis, TISCs also utilize fatty acid $\beta$ oxidation (FAO) and glutaminolysis (Figure 1) which occurs through mitochondrial respiration (11). Interesting, the stem cell features of TISCs such as cell proliferation and migration were inhibited following chemical inhibition of glycolysis, thus suggesting that the glycolytic phenotype of TISCs is needed for their efficient stem-cell functionality (12). When TISCs remain quiescent, their mitochondrial replication and metabolic activity is suppressed (13). However, when quiescent TISCs are subjected to a second-hit by mutation in oncogenes, such as a targeted mutation in a negative regulator of mammalian target of rapamycin (mTOR) complex or tuberous sclerosis complex 1 (TSC1) could lead to a colossal enhancement in the proliferation of TISCs along with upregulation in mitochondrial metabolic activity as evidenced by increase mitochondrial number per cell, elevated production of reactive oxygen species (ROS) and OXPHOS activity eventually leading to tumor relapse (14). These multiple pieces of research evidence suggest that the malignant transition of TISCs from a quiescent to a cancerous state relies on a metabolic switch from glycolytic to mitochondrialmediated OXPHOS phenotype (15). In addition, modulations in the expression of oncogenic transcription factors, such as Sox2, Oct4, c-Myc, and Klf4, also noted in NSC mediated somatic cell differentiation, are associated with the development of teratomas in murine orthotopic transplant models (16). These data suggest that there is significant overlap in the stem cell signaling mechanisms between somatic cell differentiation and carcinogenesis.

\section{UNIQUE METABOLIC CHANGES IN TISCS}

A metabolic comparison between NSCs and TISCs demonstrate that TISCs have elevated Warburg-like glycolytic metabolism with increased glucose consumption, lactate production, and ATP synthesis (17). Research in this area suggests that elevated expression of oncogenes, such as Myc expression, plays a critical role in stem cell functionality and the glycolytic metabolic footprint in some breast cancers (18). A metabolic switch from OXPHOS to glycolysis is noted in TISCs obtained from CD44+basal-like triple negative breast cancer (19). A similar shift to glycolytic metabolism was noted in CD133+TISCs obtained from radio-resistant nasopharyngeal (20) and hepatocellular carcinomas (11). Interestingly, treatment with an inhibitor of glycolysis, 3-bromopyruvate, decreased the stem cell-like functionality and made them more amenable to gemcitabine mediated cytotoxicity in aldehyde dehydrogenase $(\mathrm{ALDH})$ enriched in TISCs obtained from pancreatic ductal adenocarcinomas (21). However, in contrast, CD133+TISCs isolated from certain kinds of glioblastomas and pancreatic cancers displayed an OXPHOS metabolic preference over glycolysis for ATP synthesis (22). This metabolic switch to OXPHOS in TISCs obtained from glioblastomas was shown to be mediated by a growth factor modulating protein, IMP2, which is a known direct enhancer of gene expression in mitochondria and stem-like functionality promoting factors such as CD133, Nanog, and Oct4 (23). Similarly, a metabolic switch to OXPHOS in TISCs from pancreatic cancers was mediated by the upregulation of the transcription factor, PGC-1 $\alpha$ (peroxisome proliferatoractivated receptor- $\gamma$ coactivator- $1 \alpha)(24)$. These data suggest that while glycolysis seems to be preferred in TISCs, certain metabolic variations do occur based on the stage and type of cancer which require further study to delineate the molecular bases of these variant metabolic phenotypes.

In the tumor microenvironment, tryptophan metabolism mediated by indoleamine 2,3-dioxygenase (IDO) has a critical immunoregulatory role leading to tumor tolerance. The IDO pathway exerts an immunological role by controlling (inhibiting) inflammation, along with induction of the tolerogenic arm of adaptive immune responses in the tumor milieu. Upregulation of IDO depletes the essential amino acid tryptophan leading to activation of the stress-response kinase GCN2, which is a critical molecule involved in sensing amino acid withdrawal (25). GCN2 activation in $\mathrm{T}$ cells can inhibit their proliferation, and can skew naive CD4+T cells toward differentiation into a Treg phenotype. In addition, IDO produces the soluble factor, kynurenine, 


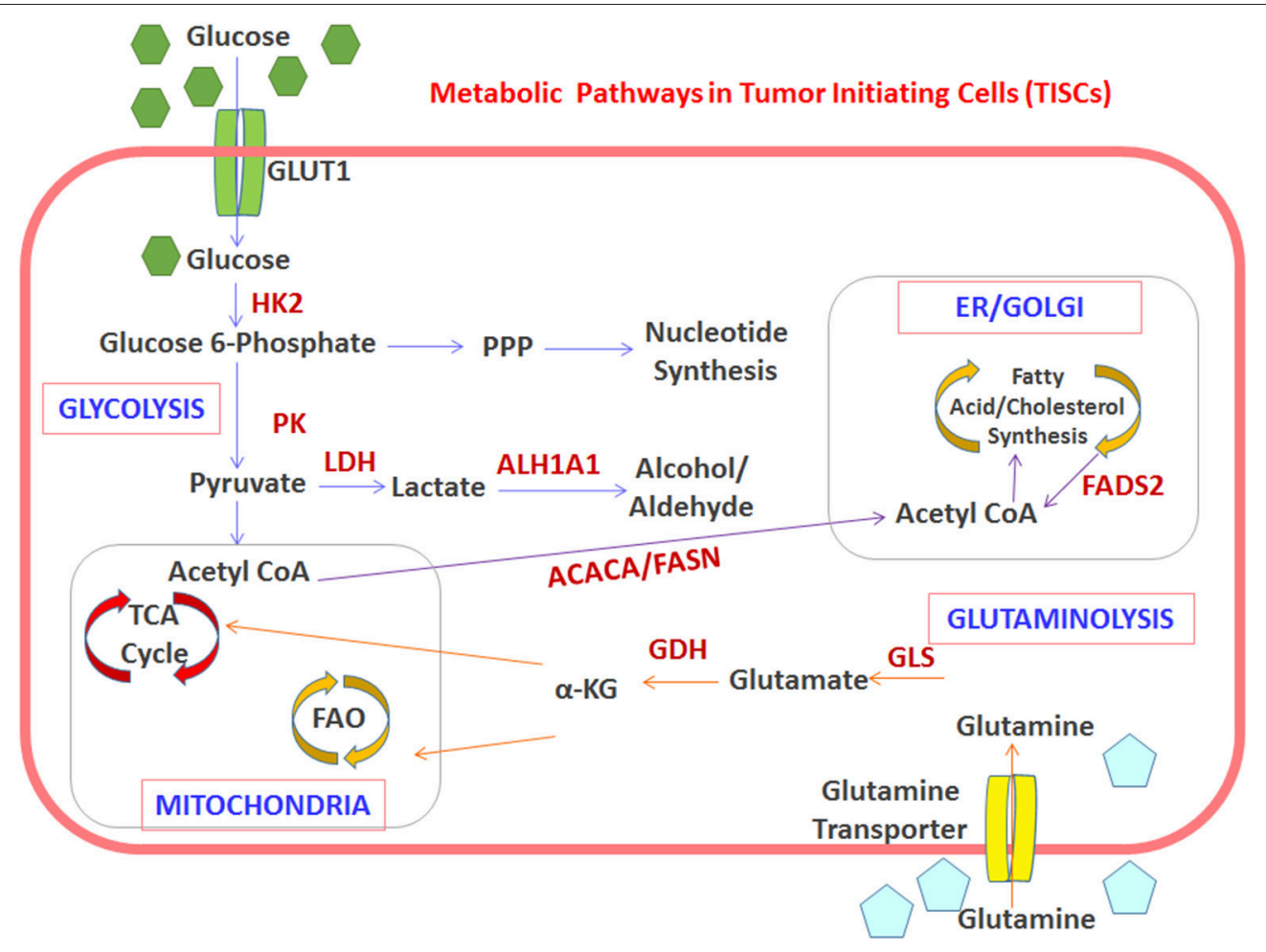

FIGURE 1 | Interplay between TISC metabolism and overexpression of potentially immunogenic antigens. The TISC-associated metabolism enhances the expression of enzymes which offer molecular targets for development of anti-TISC vaccines. Schematic representation of the metabolic switch toward OXPHOS, FA synthesis, and glutaminolysis in TISCs. Upregulated enzymes and pathways are indicated in red. HK2, hexokinase-2; PK, pyruvate kinase; GDH, glutamate dehydrogenase; GLS, glutaminase; ACACA, acetyl-CoA carboxylase; FASN, fatty acid synthase; ALDH1A1, aldehyde dehydrogenase-1A1.

which binds to and activates the aryl hydrocarbon receptor (AhR). Activation of the AhR pathway promotes Treg cell differentiation, and could also induce macrophage polarization to immunosuppressive $M \Phi 2$ phenotype. In several small animal cancer models, IDO, which is expressed by tumor cells, was found to stimulate recruitment of Tregs, resulting in the impairment of immune surveillance $(26,27)$. The use of IDO inhibitors to decrease the overall accumulation of Tregs and enhance tumor regression is an area of intense research in all solidorgan cancers. While enhanced IDO expression was noted in mesenchymal stem cells (28), a similar enhanced expression with downstream immunosuppressive effect is yet to be established in TISCs.

Fatty acid metabolism is an alternative source of energy production used by TISCs. Studies by Wang et al. showed that the lipid metabolism via fatty acid oxidation (FAO) is controlled by the JAK/ STAT3 pathway, which in turn supports breast TISCs and their resistance to conventional chemotherapy (29). These data strongly suggest that JAK/STAT3 pathway inhibition not only affects the self-renewal abilities of breast TISCs, but also blocks the expression of many genes involved in lipid metabolism, such as carnitine palmitoyl transferase 1B (CT1B). Fatty acid oxidation is also known to play a crucial role in maintaining hematopoietic stem cells (HSCs) and memory functionality of $\mathrm{CD} 8^{+} \mathrm{T}$ cells $(30)$. Therefore, FAO inhibition in HSCs has a direct negative impact on their stem cell functionality, whereas, in $\mathrm{T}$ cells, this inhibition prevents their phenotypic differentiation (31). These reports suggest that the FAO pathway prioritizes TISC survival over rapid cell proliferation and cancerous differentiation.

\section{IMPACT OF TUMOR MICROENVIRONMENT ON TISCS}

The TISCs are further regulated by pro-survival molecules released by other tumor and immune cells in the tumor microenvironment (TME). Hypoxia in the TME seems to be a strong promoter of TISC activity. Various studies have demonstrated that hypoxia in the TME induces expression of HIF- $1 \alpha$, which in turn mediates enhanced expression of downstream transcription factors with stem-like functionality such as Oct4 and Notch signaling molecules $(32,33)$. Further, high densities of cancer-associated fibroblasts (CAFs) are also known to promote tumor growth and metastasis (34). A subset of TISCs, CD44+CD90+TISCs, which have been shown to be in direct contact with CAFs in breast cancers are thought to have enhanced tumor invasive functionality (35). Similar metastatic effect was also shown by TISCs following interaction with tumor residing stromal cells in pancreatic cancers (36). Furthermore, 
in the TME, the interaction of TISCs with stromal cells is suggested to induce epithelial-mesenchymal transition (EMT) resulting in tumor proliferation and metastasis (37). Acquiring EMT properties is a well-known precursor of TISC-induction and cancerous differentiation through upregulation of common pro-cancerous signaling pathways such as the Wnt-signaling mechanism $(38,39)$. Further, on metabolic front, Wnt-signaling is shown to promote glycolysis and FAO pathways through inhibition of pyruvate dehydrogenase kinase (PDK1), along with upregulation of enzymes such as pyruvate carboxylase, alcohol dehydrogenase, acetyl-CoA carboxylase, and fatty acid synthetase, all of which are associated with the promotion of TISC-mediated EMT $(40,41)$.

Establishing blood supply to provide oxygen and nutrients is imperative to tumor growth and proliferation. This process of tumor angiogenesis is facilitated by active recruitment of proangiogenic endothelial progenitor cells (42). Research on TISCs obtained from glioblastomas have demonstrated that these TISCs have a potential to differentiate into pro-angiogenic endothelial cells potentially leading to vascularization of these tumors. Molecular mechanistic studies have revealed that enhanced expression of vascular endothelial growth factor (VEGF) by tumor cells leads to differentiation of these TISCs into endothelial progenitor cells (43). Further, the cytokine, interleukin-6 (IL-6), is known to induce vascularization in tumors. Inhibition of IL6-mediated vascular response by inhibition by IL-6 shRNA and IL-6-receptor blocking by tocilizumab demonstrated inhibited cancerous differentiation and growth by CD44+ALDH+TISCs obtained from head and neck squamous cancers (44). These observations suggest that TISCs have angiogenic potential by their ability to differentiate into pro-angiogenic endothelial cells which could be utilized as a therapeutic intervention strategy.

\section{POTENTIAL IMPACT OF TISC-METABOLIC PHENOTYPE ON INNATE IMMUNE RESPONSES}

Natural killer (NK) cells play a critical role in innate effector immune responses and tumor immune surveillance. While NK cells exert cytotoxicity of tumor cells their effect on antiTISC immune responses remains undefined. The TISCs have a paradoxical effect toward induction of immune responses. In general, stem-cells, such as TISCs and NSCs, have an inherent immunosuppressive functionality. The TISCs due to their inherent stem-cell functionality have the ability to evade cytotoxic innate and adaptive immune responses. However, in contrast, due to the expression of unique TISC-associated antigens, TISCs could be immunogenic. This intriguing paradoxical effect poses both a challenge and an opportunity toward development of TISC-based anti-cancer immunotherapeutic strategies. A recent study by Ames et al., utilizing orthotopic human tumor implants in immunodeficient murine cancer models, have suggested that NK cells due to their ability to home into non-dividing cells could preferentially target TISCs (45). The authors have utilized the TISC-associated marker
ALDH1 to sort stem cells from pancreatic, breast and sarcoma cancer cell lines. The results from this study demonstrated that NK cells co-cultured with these ALDH1 sorted cancer stem cells exerted their cytotoxicity upon ALDH1 ${ }^{\text {high }}$ cells more effectively than the ALDH1 ${ }^{\text {low }}$ ones. This preferential targeting was further confirmed on human tumor specimens in single-cell suspension and allogeneic NK cell co-treatment. Based on the molecular mechanistic studies performed in these experiments, the authors conclude that TISCs due to their enhanced surface expression of NKG2D ligands (MICA/B) were able to activate NKG2D receptors resulting in the final NK cell cytotoxic functionality against TISCs. Similar evidence was obtained from other laboratories with studies on TISCs obtained from human colorectal cancers (46). However, in contrast to the above findings, studies with CD133+ brain TISCs demonstrated no significant expression levels of NK cell activating ligands (MICA and MICB), and thus making these glioma TISCs resistant to NK cell-mediated elimination (47). Along these lines, Wang et al., have reported that, in human breast TISCs, aberrant expression of oncogenic miR-20a caused a downregulation of the expression of NKG2D ligands (MICA/B) and eventually resulted in a decreased activation of NK cell receptor NKG2D receptors resulting in possible immune escape of these breast TISCs from NK cell mediated tumor cytotoxicity (28). These data suggest that TISCs probably due to their immature cell differentiation status are not amenable to innate immune mediated tumor elimination response. Taken together, in spite of these conflicting reports, all these interesting observations could provide a strong platform for futuristic NK cell-based anti-cancer immunotherapeutic approaches to eliminate otherwise treatment-resistant TISCs. Furthermore, currently there is only a minimal understanding of the molecular correlation between ALDH1, a protein associated with TISC-associated metabolism, and expression of NKG2D ligands (MICA/B) in TISCs. Mechanistic studies in this direction could shed better light on the potential success of NK cell based immunotherapy.

The immune system due to its double-edged sword nature could exert either inhibitory (tolerance) or stimulatory (cytotoxic) responses on tumor progression. Although, macrophages, due to their phagocytic functionality, are generally considered part of the innate-arm of immunity, tumor infiltrating macrophages display unique two-dimensional plasticity to polarize into two apparently opposite phenotypes (48). While tumor infiltrating macrophages (TAMs) under the influence of various cytokines and chemokines in the TME differentiate into anti-tumor $\mathrm{M} \Phi 1$ or pro-tumor $\mathrm{M} \Phi 2$ phenotypes, it is well-recognized that most TAMs display the immunosuppressive $\mathrm{M} \Phi 2$ phenotype. Cancer cells induce a MФ2 phenotypic polarization resulting in the secretion of anti-inflammatory and pro-angiogenic cytokines such as, IL-10 and VEGF, which also drive TISC self-renewal by activating cell growth and angiogenesis-related signaling pathways (49). It has been shown that hypoxia in the tumor microenvironment preferentially upregulates OXPHOS and FAO metabolic pathways in the MФ2 TAMs $(50,51)$. This causes accumulation of metabolic bi-products such as glutamine, $\alpha$-ketoglutarate, and succinate resulting in activation of the HIF- $1 \alpha$-mediated 
cell-self-renewal signaling pathway which is also shown to be critical in TISC functionality (52). Further, TAMs have also been shown to directly enhance carcinogenesis and TISC-dependent chemoresistance through STAT3 transcription factor activation. Other studies have shown a mutually symbiotic relationship between TISCs and MФ2 TAMs, in that TISCs were considered to play an active role in $\mathrm{M} \Phi 2$ polarization resulting in inhibition of antigen presentation and anti-tumor cytotoxic CD8+T cell responses (53).

\section{TISC-ASSOCIATED ANTIGENS}

The ability of a host to recognize TISCs as non-self and mount an efficient effector immune response would be critical for the development of novel TISC based immunotherapeutic strategies. Antigen expression is essential for recognition by naïve $\mathrm{T}$ lymphocytes and efficient induction of a CD8+T lymphocyte (CTL) response (54). Unique antigen expression profiles in TISCs have been reported in several malignancies. TISC-associated antigen profiles (Figure 2) could be a result of either overexpression of antigen, expression of a differentiation antigen, or mutation of normal somatic protein resulting in neoantigens (55). As TISCs are immature forms of cancer cell differentiation, the differentiation antigens are not generally considered as suitable targets for the development of TISCassociated immunotherapeutic strategies. TISCs express several overexpressed antigens, such as CEP55, COA1 etc., which are also over-expressed in normal stem cells $(56,57)$. Although all over-expressed antigens might not be strong immunotherapeutic targets, certain other types of overexpressed antigens, such as ALDH1A1 (58), survivin, livin, and $\operatorname{Bcl}-2$ (59, 60), have been reported in TISCs. These antigens while ubiquitous and expressed in minimal quantities in normal organs, are overexpressed in TISCs and play a critical role in tumorigenesis. Along with this, organ-associated over-expression of antigens such as, hTERT in CD44+ breast cancer TISCs (61), HER2 proto-oncogene in glioma TISCs (62), CEP55, and COA-1 (63) in colon TISCs is well-established. These overexpressed antigens could be novel targets for the development of TISC-associated immunotherapeutic strategies.

Expression of neo-antigens in TISCs is a result of genomic DNA mutations resulting in the production of tumor-associated antigens $(64,65)$. As the antigenic peptide epitopes of these neo-antigens are not significantly affected by central T-cell tolerance compared with non-mutated self-antigens (Figure 3), these neo-antigens could offer attractive peptide-base anticancer vaccine strategies (66). With the advent of mass spectrometry and next-generation exome sequencing tools, neoantigens are expected to play a critical role in personalized cancer medicine $(67,68)$. However, these in silico neo-antigen identification techniques should be supplemented by extensive bench-work studies to determine if the mutation resulting in a neo-antigen is a driver mutation with cell growth advantage or passenger mutation with no cell growth advantage (Figure 4). Further, expression of neo-antigens depends upon the actual transcription and translation of the mutated gene, thus, limiting the final number of neo-antigens in TISCs and compromising the potential development of immunotherapeutic strategies (69).

Activation and differentiation of naïve CD8+T lymphocytes to cytotoxic effector cells requires efficient loading and membrane surface presentation of immunodominant epitopes by HLA class I (70). However, efficient presentation of TISCassociated antigenic peptides is reduced due to downregulation of surface expression of HLA class I molecules on mature cancer cells. This helps TISCs escape host immune surveillance. Loading of MHC class I molecules with TISC-associated antigenic peptides requires intact antigen presenting machinery (APM) comprised of the proteasome complex needed for cleavage of antigenic proteins, transport of cleaved antigenic peptides into endoplasmic reticulum (ER) through transporters associated with antigen processing-1 and-2 (TAP1 and TAP2), further intra-ER cleavage of peptides to appropriate length to be loaded on to MHC class I and $\beta 2$-microglobulin (B2M) complex by endoplasmic reticulum aminopeptidase associated with antigen processing (ERAAP), and several chaperone molecules, such as tapasin, calreticulin, ERp57, and calnexin, needed for efficient antigenic peptide loading onto MHC class I (71). In contrast to tumor cells, studies by Chikamatsu et al. report that CD44+TISCs demonstrated expression levels of the molecules involved in antigen presentation machinery (APM), such as LMP2, LMP7, TAP1 etc., equivalent to that of CD44 negative stromal non-stem cells. These data clearly suggest that TISCs have the ability to present immunodominant epitopes by HLA class I molecules and, therefore, could potentially be killed by TISC-associated antigen-specific cytotoxic response by CD8+T lymphocytes (CTL) (72). Interestingly, HER2-specific peptide vaccination reduced ALDH positive breast cancer associated TISCs in MMTV-PyMT murine transgenic breast cancer models, suggesting a potential immunotherapeutic treatment strategy against treatment resistant TISCs (73). However, there seems to be varying evidence for the expression of HLA class I molecules on TISCs based on the tissue origin. For example, studies on TISCs derived from melanoma demonstrated reduced HLA class I expression suggesting that HLA class I expression in TISCs depends on the tumor type (74). Furthermore, even within the same tumor type, two apparently conflicting reports came from two different groups. While one research group showed enhanced HLA class I expression in glioblastoma multiform (GBM) derived TISCs isolated as sphere-forming cells, while other research group working on similar GBM derived TISCs as sphere-forming cells reported lower HLA class I expression (75). Further, analysis on in vitro 3D cell cultures of TISCs derived from GBM has demonstrated reduced expression of immuno-stimulatory CD80 and CD86 molecules, while, there was an enhanced expression of the immuno-inhibitory molecule, PD-L1, and secretion of the immunosuppressive cytokine, IL-10 (76). This immunosuppressive profile of TISCs might contribute to creating an immune-suppressive tumor microenvironment resulting in tumor immune escape. The difference in HLA expression and ability to mount adaptive immune responses amongst various cancer cell lines could be from a difference in TISCs isolation methodology and/or 


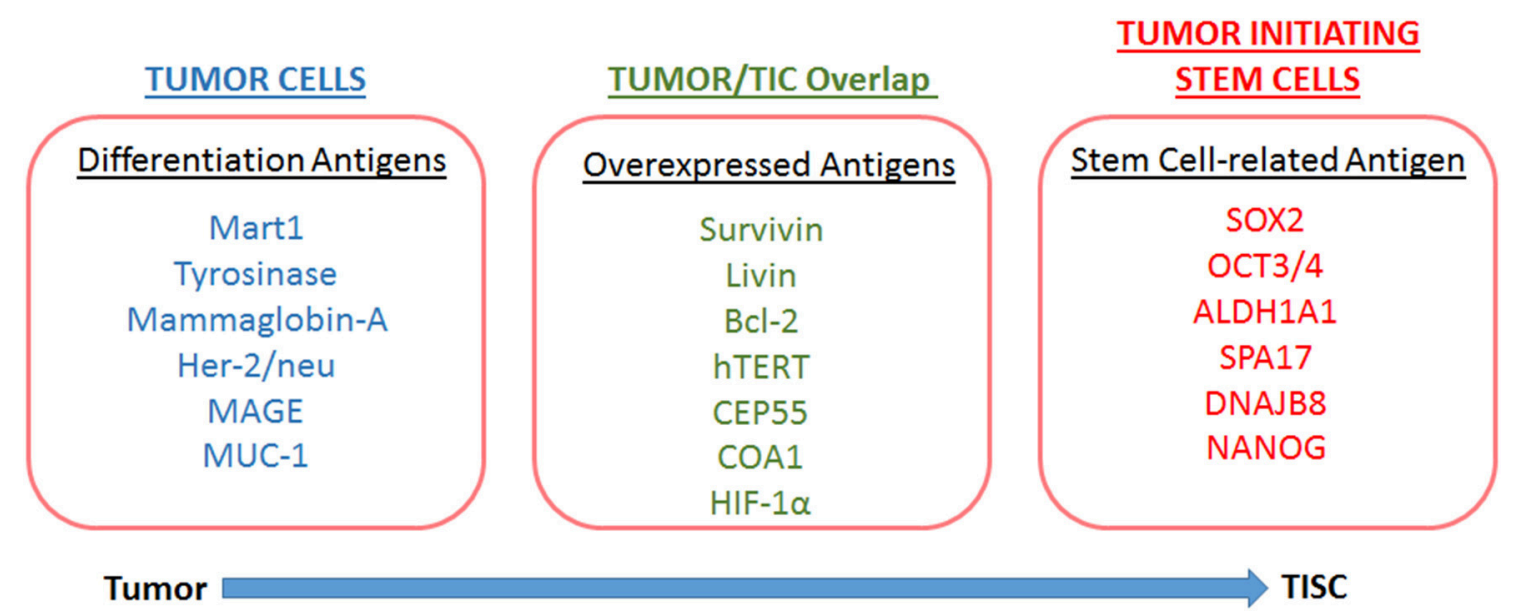

FIGURE 2 | Overview of the antigens associated to tumors and tumor-initiating stem cells.

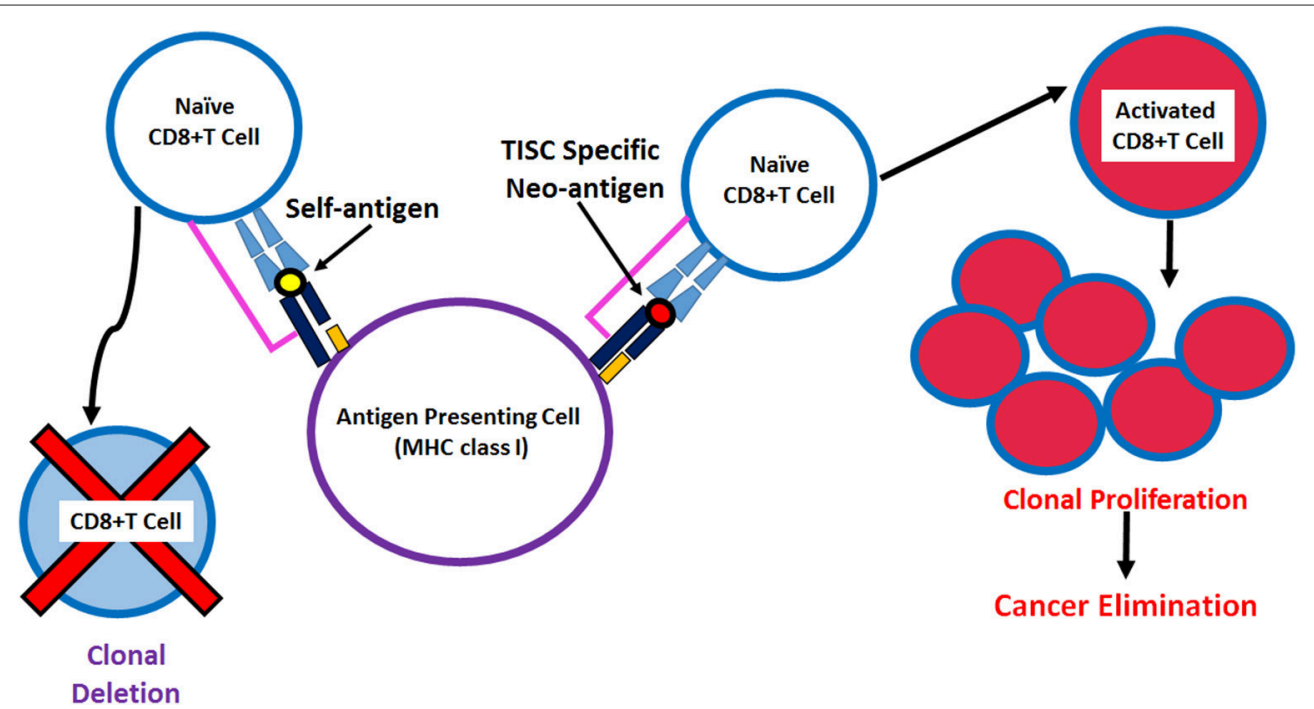

FIGURE 3 | Schematic representation of the antigen presentation of neoantigen derived immunodominant epitopes by MHC class I molecules to activate CD8+T cell against tumor initiating stem cells.

varying organ-associated origin of TISCs which requires further investigation.

\section{TISC ANTIGEN-BASED VACCINES AND MONOCLONAL ANTIBODIES}

The enzymes overexpressed to mediate TISC-skewed metabolic pathways are considered to be good antigenic targets for development of anti-TISC-based immunotherapies. For example, an isoform of aldehyde dehydrogenase (ALDH1A1) promotes cell-survival by reducing the intracellular cytotoxic oxidative damage mediated by oxidation of aldehydes to carboxylic acids (77). Enhanced ALDH1A1 enzymatic activity was noted in TISCs obtained from various solid organ tumors. In addition, ALDH1A1 is considered to detoxify metabolic biproducts from chemotherapy and thus conferring cancer resistance in ALDH1A1 $1^{\text {high }}$ cancer cells (78). Dylla et al., have demonstrated that shRNA based knock-down of ALDH1A1 mRNA significantly increased the chemo-susceptibility of TISCs obtained from colon cancer to cyclophosphamide therapy (79). Several groups have demonstrated that ALDH is an attractive antigenic target for induction of anti-cancer adaptive immune responses. Visus et al. have utilized immunodominant epitopes derived from ALDH to generate cytotoxic effector CD8+T cells specifically against ALDH producing cancer cells (35, 58). Interestingly, autologous dendritic cells pulsed by immunodominant epitopes derived from ALDH were utilized for in vitro stimulation of patients' $\mathrm{CD} 8+\mathrm{T}$ cells to activate them into cytotoxic cells. Similarly, in preclinical immunocompromised 


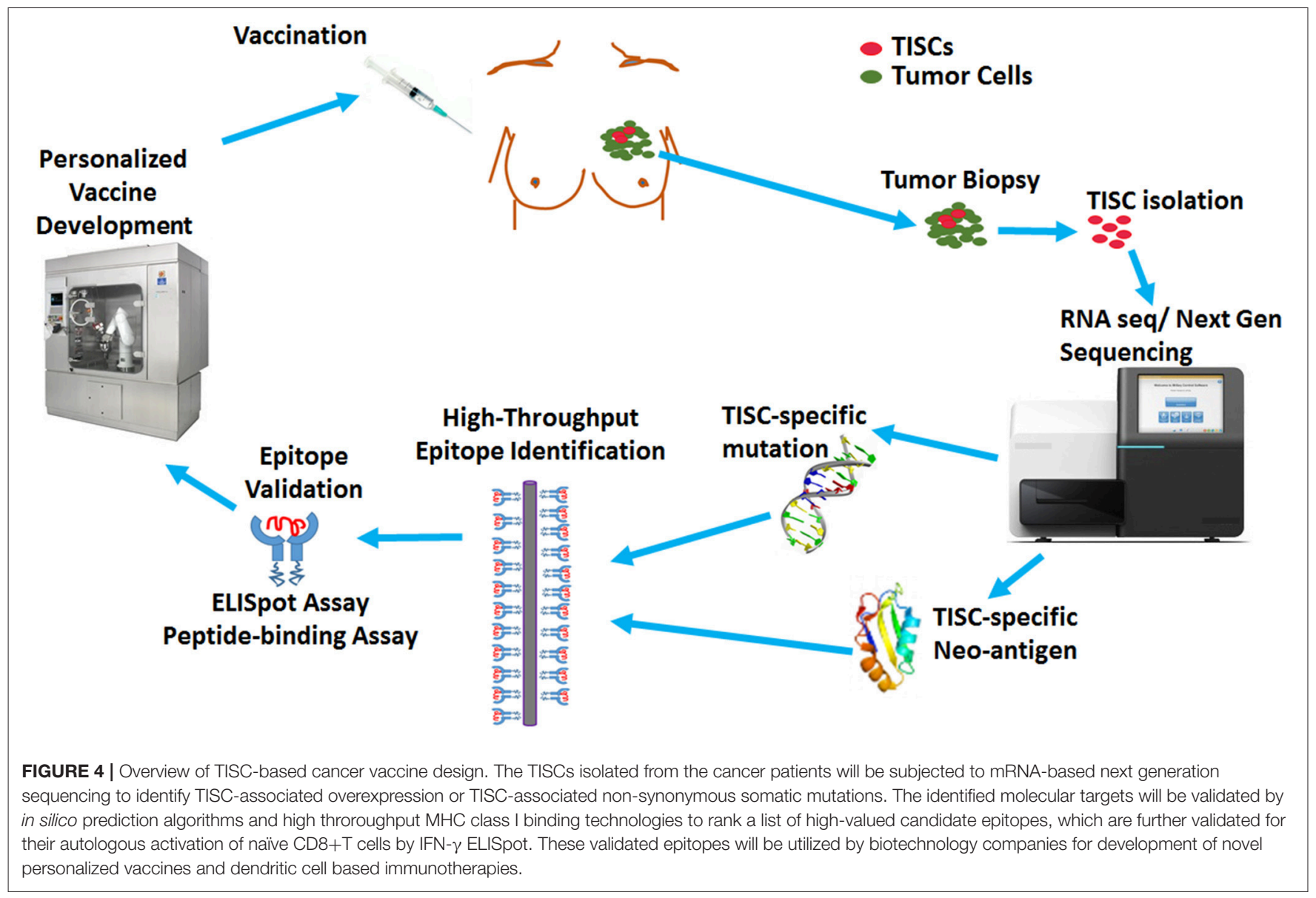

murine cancer models adoptive transfer of CD8+T cells activated by ALDH-epitope pulsed dendritic cells reduced the tumor growth kinetics of human cancer xenografts (80). Taken together, these data provide compelling evidence for the future utilization of ALDH as TISC-associated antigenic target to develop novel peptide and DNA-based vaccine strategies. Further, in vitro activation of dendritic cells (DCs), by antigenic epitopes and eventual adoptive transfer of these DCs is considered a promising anti-cancer vaccination strategy. Phuc and colleagues reported that breast TISC-associated antigen derived DC vaccine could lead to migration of adoptively transferred pulsedDCs to the spleen and activation of naive CD8+T cells and induce anti-tumor cytotoxicity (81). These observations, although require further study, nevertheless, strongly suggest the futuristic application of TISC-associated antigen pulsed DCbased immunotherapy.

A TISC-associated membrane protein, CD44, is a $90 \mathrm{kDa}$ glycosylated type-1 p-glycoprotein which is involved in several stem cell-like functions such as self-renewal, cell division, antiapoptosis, and distant metastasis (82). Targeting TISCs by inhibiting CD44 signaling with blocking monoclonal antibodies $(\mathrm{mAb})$ has emerged as a promising anti-cancer therapy. Seiter et al., have shown a reduced proliferation and lung metastases of pancreatic adenocarcinoma in small animal tumor model following treatment with $\mathrm{mAbs}$ against $\mathrm{CD} 44 \mathrm{v}$, a splice variant
ofCD44 (83). Following a similar approach, Jin et al., have demonstrated that treatment with CD44 mAb specifically eradicated AML TISCs in immunodeficient murine cancer model (84). Further, there was an $80 \%$ reduction of BxPC3 pancreatic tumor xenografts following administration of a humanized CD44-specific mAb (85). Human clinical trials with anti-CD44 $\mathrm{mAb}$ seem like a promising immunotherapeutic approach (86). Similarly, other surface markers such as CD133, survinin, Her2 , and CT proteins are targeted by mAbs to promote tumor regression in various preclinical models.

\section{CONCLUSION}

Several TISC-based immunotherapeutic approaches are under development in various stages of preclinical studies. As outlined in this review article, a careful and more exhaustive genetic and metabolic understanding of TISC-associated phenotypes is critical to develop novel TISC based immunotherapies. Various components within the tumor microenvironment such as tumor cells, infiltrating immune cells, and supporting stromal cells impact the TISC metabolism. This unique metabolic profile leads to upregulation of certain enzymes and proteins such as ALDH1, CEP55, IDO COA1 etc., which can be utilized for development of vaccine based anti-cancer immunotherapy. Further investigation 
of TISCs and the immunosuppressive phenotype caused by the tumor microenvironment will provide opportunities to not only achieve a more comprehensive understanding of tumor biology but also develop specific medical therapies to target the weaknesses underlying tumor development and attack tumor cells in more effective ways. For example, various preclinical studies have clearly demonstrated that combination immunotherapies such as vaccines, Treg depletion, or immune checkpoint blockade, together with chemotherapy have more profound outcomes compared to conventional chemotherapy alone. Further, application of novel technologies and highthroughput platforms would be needed to identify TISCassociated neoantigens for future development of anti-cancer

\section{REFERENCES}

1. Prieto-Vila M, Takahashi RU, Usuba W, Kohama I, Ochiya T. Drug resistance driven by cancer stem cells and their niche. Int J Mol Sci. (2017). 18:E2574. doi: $10.3390 /$ ijms 18122574

2. Plaks V, Kong N, Werb Z. The cancer stem cell niche: how essential is the niche in regulating stemness of tumor cells? Cell Stem Cell. (2015) 16:225-38. doi: 10.1016/j.stem.2015.02.015

3. Gerdes MJ, Sood A, Sevinsky C, Pris AD, Zavodszky MI, Ginty F. Emerging understanding of multiscale tumor heterogeneity. Front Oncol. (2014) 4:366. doi: 10.3389/fonc.2014.00366

4. Nowell PC. The clonal evolution of tumor cell populations. Science. (1976) 194:23-8. doi: 10.1126/science.959840

5. Shackleton M, Quintana E, Fearon ER, Morrison SJ. Heterogeneity in cancer: cancer stem cells versus clonal evolution. Cell. (2009) 138:822-9. doi: 10.1016/j.cell.2009.08.017

6. Hu Y, Fu L. Targeting cancer stem cells: a new therapy to cure cancer patients. Am J Cancer Res. (2012) 2:340-56.

7. Porporato PE, Filigheddu N, Pedro JMB, Kroemer G, Galluzzi L. Mitochondrial metabolism and cancer. Cell Res. (2018) 28:265-80. doi: $10.1038 /$ cr.2017.155

8. Folmes CD, Dzeja PP, Nelson TJ, Terzic A. Metabolic plasticity in stem cell homeostasis and differentiation. Cell Stem Cell. (2012) 11:596-606. doi: 10.1016/j.stem.2012.10.002

9. De Francesco EM, Sotgia F, Lisanti MP. Cancer stem cells (CSCs): metabolic strategies for their identification and eradication. Biochem J. (2018) 475:161134. doi: 10.1042/BCJ20170164

10. Ahmed N, Escalona R, Leung D, Chan E, Kannourakis G. Tumour microenvironment and metabolic plasticity in cancer and cancer stem cells: perspectives on metabolic and immune regulatory signatures in chemoresistant ovarian cancer stem cells. Semin Cancer Biol. (2018) 53:26581. doi: 10.1016/j.semcancer.2018.10.002

11. Sancho P, Barneda D, Heeschen C. Hallmarks of cancer stem cell metabolism. Br J Cancer. (2016) 114:1305-12. doi: 10.1038/bjc.2016.152

12. Martinez-Outschoorn UE, Peiris-Pages M, Pestell RG, Sotgia F, Lisanti MP. Cancer metabolism: a therapeutic perspective. Nat Rev Clin Oncol. (2017) 14:11-31. doi: 10.1038/nrclinonc.2016.60

13. Jang YY, Sharkis SJ. A low level of reactive oxygen species selects for primitive hematopoietic stem cells that may reside in the low-oxygenic niche. Blood. (2007) 110:3056-63. doi: 10.1182/blood-2007-05-087759

14. Liakou CI, Kamat A, Tang DN, Chen H, Sun J, Troncoso P, et al. CTLA-4 blockade increases IFNgamma-producing CD4+ICOShi cells to shift the ratio of effector to regulatory T cells in cancer patients. Proc Natl Acad Sci USA. (2008) 105:14987-92. doi: 10.1073/pnas.0806075105

15. Schoenhals M, Kassambara A, De Vos J, Hose D, Moreaux J, Klein B. Embryonic stem cell markers expression in cancers. Biochem Biophys Res Commun. (2009) 383:157-62. doi: 10.1016/j.bbrc.2009.02.156

16. Simsek T, Kocabas F, Zheng J, Deberardinis RJ, Mahmoud AI, Olson $\mathrm{EN}$, et al. The distinct metabolic profile of hematopoietic stem cells vaccine strategies. These TISC-associated strategies could also be combined with other immunotherapies such as immunecheck-point inhibitor (PD1/CTLA-4) for enhanced anticancer therapeutic success.

\section{AUTHOR CONTRIBUTIONS}

DK, SA, and VT participated in manuscript drafting, and revision of this review article.

\section{FUNDING}

This work was supported by NIH-5U54CA163066-6611 (VT). reflects their location in a hypoxic niche. Cell Stem Cell. (2010) 7:380-90. doi: 10.1016/j.stem.2010.07.011

17. Vermeulen L, de Sousa e Melo F, Richel DJ, Medema JP. The developing cancer stem-cell model: clinical challenges and opportunities. Lancet Oncol. (2012). 13:e83-9. doi: 10.1016/S1470-2045(11)70257-1

18. Gabay M, Li Y, Felsher DW. MYC activation is a hallmark of cancer initiation and maintenance. Cold Spring Harb Perspect Med. (2014). 4:a014241. doi: 10.1101/cshperspect.a014241

19. Dong C, Yuan T, Wu Y, Wang Y, Fan TW, Miriyala S, et al. Loss of FBP1 by Snail-mediated repression provides metabolic advantages in basal-like breast cancer. Cancer Cell. (2013) 23:316-31. doi: 10.1016/j.ccr.2013.01.022

20. Shen X, Xiong GL, Jing Y, Xiao H, Cui Y, Zhang YF, et al. The protein kinase $\mathrm{C}$ agonist prostratin induces differentiation of human myeloid leukemia cells and enhances cellular differentiation by chemotherapeutic agents. Cancer Lett. (2015) 356:686-96. doi: 10.1016/j.canlet.2014.10.018

21. Isayev O, Rausch V, Bauer N, Liu L, Fan P, Zhang Y, et al. Inhibition of glucose turnover by 3-bromopyruvate counteracts pancreatic cancer stem cell features and sensitizes cells to gemcitabine. Oncotarget. (2014) 5:5177-89. doi: 10.18632/oncotarget.2120

22. Vlashi E, Lagadec C, Vergnes L, Matsutani T, Masui K, Poulou M, et al. Metabolic state of glioma stem cells and nontumorigenic cells. Proc Natl Acad Sci USA. (2011) 108:16062-7. doi: 10.1073/pnas.1106704108

23. Janiszewska M, Suva ML, Riggi N, Houtkooper RH, Auwerx J, ClementSchatlo V, et al. Imp2 controls oxidative phosphorylation and is crucial for preserving glioblastoma cancer stem cells. Genes Dev. (2012) 26:1926-44. doi: 10.1101/gad.188292.112

24. Sancho P, Burgos-Ramos E, Tavera A, Bou Kheir T, Jagust P, Schoenhals M, et al. MYC/PGC-1alpha balance determines the metabolic phenotype and plasticity of pancreatic cancer stem cells. Cell Metab. (2015) 22:590-605. doi: 10.1016/j.cmet.2015.08.015

25. Munn DH, Mellor AL. Indoleamine 2,3 dioxygenase and metabolic control of immune responses. Trends Immunol. (2013) 34:137-43. doi: 10.1016/j.it.2012.10.001

26. Munn DH. Indoleamine 2,3-dioxygenase, Tregs and cancer. Curr Med Chem. (2011) 18:2240-6. doi: 10.2174/092986711795656045

27. Wainwright DA, Balyasnikova IV, Chang AL, Ahmed AU, Moon KS, Auffinger $\mathrm{B}$, et al. IDO expression in brain tumors increases the recruitment of regulatory T cells and negatively impacts survival. Clin Cancer Res. (2012) 18:6110-21. doi: 10.1158/1078-0432.CCR-12-2130

28. Ling W, Zhang J, Yuan Z, Ren G, Zhang L, Chen X, et al. Mesenchymal stem cells use IDO to regulate immunity in tumor microenvironment. Cancer Res. (2014) 74:1576-87. doi: 10.1158/0008-5472.CAN-13-1656

29. Wang T, Fahrmann JF, Lee H, Li YJ, Tripathi SC, Yue C, et al. JAK/STAT3regulated fatty acid beta-oxidation is critical for breast cancer stem cell self-renewal and chemoresistance. Cell Metab. (2018) 27:136-50 e135. doi: 10.1016/j.cmet.2017.11.001

30. Ito K, Carracedo A, Weiss D, Arai F, Ala U, Avigan DE, et al. A PMLPPAR-delta pathway for fatty acid oxidation regulates hematopoietic stem cell maintenance. Nat Med. (2012) 18:1350-8. doi: 10.1038/nm.2882 
31. Lugli E, Dominguez MH, Gattinoni L, Chattopadhyay PK, Bolton DL, Song $\mathrm{K}$, et al. Superior $\mathrm{T}$ memory stem cell persistence supports long-lived $\mathrm{T}$ cell memory. J Clin Invest. (2013) 123:594-99. doi: 10.1172/JCI66327

32. Demaria M, Giorgi C, Lebiedzinska M, Esposito G, D’Angeli L, Bartoli A, et al. A STAT3-mediated metabolic switch is involved in tumour transformation and STAT3 addiction. Aging. (2010) 2:823-42. doi: 10.18632/aging.1 00232

33. Li Z, Bao S, Wu Q, Wang H, Eyler C, Sathornsumetee S, et al. Hypoxiainducible factors regulate tumorigenic capacity of glioma stem cells. Cancer Cell. (2009) 15:501-13. doi: 10.1016/j.ccr.2009.03.018

34. Vermeulen L, De Sousa EMF, van der Heijden M, Cameron K, de Jong $\mathrm{JH}$, Borovski T, et al. Wnt activity defines colon cancer stem cells and is regulated by the microenvironment. Nat Cell Biol. (2010) 12:468-76. doi: $10.1038 /$ ncb2048

35. Visus C, Ito D, Amoscato A, Maciejewska-Franczak M, Abdelsalem A, Dhir R, et al. Identification of human aldehyde dehydrogenase 1 family member $\mathrm{A} 1$ as a novel $\mathrm{CD} 8+\mathrm{T}$-cell-defined tumor antigen in squamous cell carcinoma of the head and neck. Cancer Res. (2007) 67:10538-45. doi: 10.1158/0008-5472.CAN-07-1346

36. Lonardo E, Frias-Aldeguer J, Hermann PC, Heeschen C. Pancreatic stellate cells form a niche for cancer stem cells and promote their self-renewal and invasiveness. Cell Cycle. (2012) 11:1282-90. doi: 10.4161/cc.19679

37. Huang CH, Peng S, He L, Tsai YC, Boyd DA, Hansen TH, et al. Cancer immunotherapy using a DNA vaccine encoding a single-chain trimer of MHC class I linked to an HPV-16 E6 immunodominant CTL epitope. Gene Ther. (2005) 12:1180-6. doi: 10.1038/sj.gt.3302519

38. Lee M, Yoon JH. Metabolic interplay between glycolysis and mitochondrial oxidation: the reverse Warburg effect and its therapeutic implication. World J Biol Chem. (2015) 6:148-61. doi: 10.4331/wjbc.v6.i3.148

39. Seok H, Noh JY, Lee DY, Kim SJ, Song CS, Kim YC. Effective humoral immune response from a H1N1 DNA vaccine delivered to the skin by microneedles coated with PLGA-based cationic nanoparticles. J Control Release. (2017) 265:66-74. doi: 10.1016/j.jconrel.2017.04.027

40. Sherwood V. WNT signaling: an emerging mediator of cancer cell metabolism? Mol Cell Biol. (2015) 35:2-10. doi: 10.1128/MCB.00992-14

41. Aspuria PP, Lunt SY, Varemo L, Vergnes L, Gozo M, Beach JA, et al. Succinate dehydrogenase inhibition leads to epithelial-mesenchymal transition and reprogrammed carbon metabolism. Cancer Metab. (2014) 2:21. doi: 10.1186/2049-3002-2-21

42. De Bock K, Georgiadou M, Carmeliet P. Role of endothelial cell metabolism in vessel sprouting. Cell Metab. (2013) 18:634-47. doi: 10.1016/j.cmet.2013.08.001

43. Ricci-Vitiani L, Pallini R, Biffoni M, Todaro M, Invernici G, Cenci T, et al. Tumour vascularization via endothelial differentiation of glioblastoma stemlike cells. Nature. (2010) 468:824-8. doi: 10.1038/nature09557

44. Krishnamurthy S, Warner KA, Dong Z, Imai A, Nor C, Ward BB, et al. Endothelial interleukin-6 defines the tumorigenic potential of primary human cancer stem cells. Stem Cells. (2014) 32:2845-57. doi: 10.1002/stem.1793

45. Ames E, Canter RJ, Grossenbacher SK, Mac S, Chen M, Smith RC, et al. NK cells preferentially target tumor cells with a cancer stem cell phenotype. $J$ Immunol. (2015) 195:4010-9. doi: 10.4049/jimmunol.1500447

46. Tallerico R, Todaro M, Di Franco S, Maccalli C, Garofalo C, Sottile R, et al. Human NK cells selective targeting of colon cancer-initiating cells: a role for natural cytotoxicity receptors and MHC class I molecules. J Immunol. (2013) 190:2381-90. doi: 10.4049/jimmunol.1201542

47. Castriconi R, Daga A, Dondero A, Zona G, Poliani PL, Melotti A, et al. NK cells recognize and kill human glioblastoma cells with stem cell-like properties. J Immunol. (2009) 182:3530-9. doi: 10.4049/jimmunol.0802845

48. Guo Q, Jin Z, Yuan Y, Liu R, Xu T, Wei H, et al. New mechanisms of tumorassociated macrophages on promoting tumor progression: recent research advances and potential targets for tumor immunotherapy. I Immunol Res. (2016) 2016:9720912. doi: 10.1155/2016/9720912

49. Krstic J, Trivanovic D, Jaukovic A, Santibanez JF, Bugarski D. Metabolic plasticity of stem cells and macrophages in cancer. Front Immunol. (2017) 8:939. doi: 10.3389/fimmu.2017.00939

50. O'Neill LA, Pearce EJ. Immunometabolism governs dendritic cell and macrophage function. J Exp Med. (2016) 213:15-23. doi: $10.1084 /$ jem. 20151570
51. Jha AK, Huang SC, Sergushichev A, Lampropoulou V, Ivanova Y, et al. Network integration of parallel metabolic and transcriptional data reveals metabolic modules that regulate macrophage polarization. Immunity. (2015) 42:419-30. doi: 10.1016/j.immuni.2015.02.005

52. Tannahill GM, Curtis AM, Adamik J, Palsson-McDermott EM, McGettrick AF, Goel G, et al. Succinate is an inflammatory signal that induces IL-1beta through HIF-1alpha. Nature. (2013) 496:238-42. doi: 10.1038/nature11986

53. Ginestier C, Liu S, Diebel ME, Korkaya H, Luo M, Brown M, et al. CXCR1 blockade selectively targets human breast cancer stem cells in vitro and in xenografts. J Clin Invest. (2010) 120:485-97. doi: 10.1172/JCI39397

54. Hirohashi Y, Torigoe T, Tsukahara T, Kanaseki T, Kochin V, Sato N. Immune responses to human cancer stem-like cells/cancer-initiating cells. Cancer Sci. (2016) 107:12-7. doi: 10.1111/cas.12830

55. Coulie PG, Van den Eynde BJ, van der Bruggen P, Boon T. Tumour antigens recognized by T lymphocytes: at the core of cancer immunotherapy. Nat Rev Cancer. (2014) 14:135-46. doi: 10.1038/nrc3670

56. Nakatsugawa M, Takahashi A, Hirohashi Y, Torigoe T, Inoda S, Murase $\mathrm{M}$, et al. SOX2 is overexpressed in stem-like cells of human lung adenocarcinoma and augments the tumorigenicity. Lab Invest. (2011) 91:1796-804. doi: 10.1038/labinvest.2011.140

57. Dhodapkar KM, Feldman D, Matthews P, Radfar S, Pickering R, Turkula S, et al. Natural immunity to pluripotency antigen OCT4 in humans. Proc Natl Acad Sci USA. (2010) 107:8718-23. doi: 10.1073/pnas.0915086107

58. Visus C, Wang Y, Lozano-Leon A, Ferris RL, Silver S, Szczepanski MJ, et al. Targeting ALDH(bright) human carcinoma-initiating cells with ALDH1A1-specific CD8(+) T cells. Clin Cancer Res. (2011) 17:6174-84. doi: 10.1158/1078-0432.CCR-11-1111

59. Madjd Z, Mehrjerdi AZ, Sharifi AM, Molanaei S, Shahzadi SZ, Asadi-Lari M. $\mathrm{CD} 44+$ cancer cells express higher levels of the anti-apoptotic protein Bcl-2 in breast tumours. Cancer Immun. (2009) 9:4.

60. Jin F, Zhao L, Zhao HY, Guo SG, Feng J, Jiang XB, et al. Comparison between cells and cancer stem-like cells isolated from glioblastoma and astrocytoma on expression of anti-apoptotic and multidrug resistance-associated protein genes. Neuroscience. (2008) 154:541-50. doi: 10.1016/j.neuroscience.2008.03.054

61. Chung SS, Aroh C, Vadgama JV. Constitutive activation of STAT3 signaling regulates hTERT and promotes stem cell-like traits in human breast cancer cells. PLoS ONE. (2013) 8:e83971. doi: 10.1371/journal.pone.0083971

62. Ahmed N, Salsman VS, Kew Y, Shaffer D, Powell S, Zhang YJ, et al. HER2specific $\mathrm{T}$ cells target primary glioblastoma stem cells and induce regression of autologous experimental tumors. Clin Cancer Res. (2010) 16:474-85. doi: 10.1158/1078-0432.CCR-09-1322

63. Inoda S, Hirohashi Y, Torigoe T, Morita R, Takahashi A, Asanuma H, et al. Cytotoxic T lymphocytes efficiently recognize human colon cancer stem-like cells. Am J Pathol. (2011) 178:1805-13. doi: 10.1016/j.ajpath.2011.01.004

64. Schumacher TN, Schreiber RD. Neoantigens in cancer immunotherapy. Science. (2015) 348:69-74. doi: 10.1126/science.aaa4971

65. Pan Q, Li Q, Liu S, Ning N, Zhang X, Xu Y, et al. Concise review: targeting cancer stem cells using immunologic approaches. Stem Cells. (2015) 33:208592. doi: 10.1002/stem.2039

66. Amara S, Tiriveedhi V. The five immune forces impacting DNAbased cancer immunotherapeutic strategy. Int J Mol Sci. (2017) 18:650. doi: 10.3390/ijms18030650

67. Yadav M, Jhunjhunwala S, Phung QT, Lupardus P, Tanguay J, Bumbaca S, et al. Predicting immunogenic tumour mutations by combining mass spectrometry and exome sequencing. Nature. (2014) 515:572-6. doi: 10.1038/nature14001

68. Tiriveedhi V. Impact of precision medicine on drug repositioning and pricing: a too small to thrive crisis. J Pers Med. (2018) 8:1-11. doi: 10.3390/jpm8040036

69. Lee CH, Yelensky R, Jooss K, Chan TA. Update on tumor neoantigens and their utility: why it is good to be different. Trends Immunol. (2018) 39:536-48. doi: 10.1016/j.it.2018.04.005

70. Aptsiauri N, Cabrera T, Garcia-Lora A, Lopez-Nevot MA, Ruiz-Cabello F, Garrido F. MHC class I antigens and immune surveillance in transformed cells. Int Rev Cytol. (2007) 256:139-89. doi: 10.1016/S0074-7696(07)56005-5

71. Blum JS, Wearsch PA, Cresswell P. Pathways of antigen processing. Annu Rev Immunol. (2013) 31:443-73. doi: 10.1146/annurev-immunol-032712-095910

72. Chikamatsu K, Takahashi G, Sakakura K, Ferrone S, Masuyama K. Immunoregulatory properties of $\mathrm{CD} 44+$ cancer stem-like cells in squamous 
cell carcinoma of the head and neck. Head Neck. (2011) 33:208-15. doi: 10.1002/hed.21420

73. Gil EY, Jo UH, Lee HJ, Kang J, Seo JH, Lee ES, et al. Vaccination with ErbB-2 peptides prevents cancer stem cell expansion and suppresses the development of spontaneous tumors in MMTV-PyMT transgenic mice. Breast Cancer Res Treat. (2014) 147:69-80. doi: 10.1007/s10549-014-3086-4

74. Schatton T, Schutte U, Frank NY, Zhan Q, Hoerning A, Robles SC, et al. Modulation of T-cell activation by malignant melanoma initiating cells. Cancer Res. (2010) 70:697-708. doi: 10.1158/0008-5472.CAN-09-1592

75. Wei J, Barr J, Kong LY, Wang Y, Wu A, Sharma AK, et al. Glioma-associated cancer-initiating cells induce immunosuppression. Clin Cancer Res. (2010) 16:461-73. doi: 10.1158/1078-0432.CCR-09-1983

76. Yao $\mathrm{Y}$, Wang $\mathrm{X}$, Jin $\mathrm{K}$, Zhu J, Wang $\mathrm{Y}$, Xiong S, et al. B7-H4 is preferentially expressed in non-dividing brain tumor cells and in a subset of brain tumor stem-like cells. J Neurooncol. (2008) 89:121-9. doi: 10.1007/s11060-008-9601-x

77. Tomita H, Tanaka K, Tanaka T, Hara A. Aldehyde dehydrogenase $1 \mathrm{~A} 1$ in stem cells and cancer. Oncotarget. (2016) 7:11018-32. doi: 10.18632/oncotarget.6920

78. Clark DW, Palle K. Aldehyde dehydrogenases in cancer stem cells: potential as therapeutic targets. Ann Transl Med. (2016) 4:518. doi: $10.21037 /$ atm.2016.11.82

79. Dylla SJ, Beviglia L, Park IK, Chartier C, Raval J, Ngan L, et al. Colorectal cancer stem cells are enriched in xenogeneic tumors following chemotherapy. PLoS ONE. (2008) 3:e2428. doi: 10.1371/journal.pone. 0002428

80. Xu Q, Liu G, Yuan X, Xu M, Wang H, Ji J, et al. Antigen-specific T-cell response from dendritic cell vaccination using cancer stem-like cell-associated antigens. Stem Cells. (2009) 27:1734-40. doi: 10.1002/stem.102

81. Phuc PV, Nguyet NTM, ThuYDT, Dong LV, Kiet TD, Ngoc PK. Effect of breast cancer stem cell extract primed dendritic cell transplantation on breast cancer tumor murine models. Ann Rev Res Biol. (2011) 1:1-13. doi: 10.2147/OTT.S77554

82. Todaro M, Gaggianesi M, Catalano V, Benfante A, Iovino F, Biffoni M, et al. CD44v6 is a marker of constitutive and reprogrammed cancer stem cells driving colon cancer metastasis. Cell Stem Cell. (2014) 14:342-56. doi: 10.1016/j.stem.2014.01.009

83. Seiter S, Arch R, Reber S, Komitowski D, Hofmann M, Ponta H, et al. Prevention of tumor metastasis formation by anti-variant CD44. J Exp Med. (1993) 177:443-55. doi: 10.1084/jem.177.2.443

84. Jin L, Hope KJ, Zhai Q, Smadja-Joffe F, Dick JE. Targeting of CD44 eradicates human acute myeloid leukemic stem cells. Nat Med. (2006) 12:1167-74. doi: $10.1038 / \mathrm{nm} 1483$

85. Zhao S, Chen C, Chang K, Karnad A, Jagirdar J, Kumar AP, et al. CD44 expression level and isoform contributes to pancreatic cancer cell plasticity, invasiveness, and response to therapy. Clin Cancer Res. (2016) 22:5592-604. doi: 10.1158/1078-0432.CCR-15-3115

86. Molejon MI, Tellechea JI, Moutardier V, Gasmi M, Ouaissi M, Turrini O, et al. Targeting CD44 as a novel therapeutic approach for treating pancreatic cancer recurrence. Oncoscience. (2015) 2:572-5. doi: 10.18632/oncoscience.172

Conflict of Interest Statement: The authors declare that the research was conducted in the absence of any commercial or financial relationships that could be construed as a potential conflict of interest.

Copyright $(\odot) 2019$ Khandekar, Amara and Tiriveedhi. This is an open-access article distributed under the terms of the Creative Commons Attribution License (CC BY). The use, distribution or reproduction in other forums is permitted, provided the original author(s) and the copyright owner(s) are credited and that the original publication in this journal is cited, in accordance with accepted academic practice. No use, distribution or reproduction is permitted which does not comply with these terms. 